\title{
Frequência de enteroparasitas e condições socioeconômicas de escolares da cidade de São Marcos-RS
}

\section{Frequency of intestinal parasites and socioeconomic conditions of São Marcos city-RS schoolchildren}

\author{
Scheila Cristina Rech ${ }^{1}$, Natália Inês Cavagnolli², Patrícia Kelly Wilmsen Dalla \\ Santa Spada ${ }^{3}$, Adriana Dalpicolli Rodrigues ${ }^{4}$
}

\begin{abstract}
Resumo
O presente estudo teve o objetivo de avaliar a frequência de parasitas intestinais em escolares do ensino fundamental do município de São Marcos-RS, no período de março a outubro de 2015. Aplicou-se um questionário sobre conhecimentos e meios de transmissão de parasitoses e sobre condições de saneamento básico da cidade; e foram analisadas amostras de fezes, processadas por sedimentação espontânea, de estudantes da pré-escola a oitava série, de nove escolas da cidade. Dos 190 escolares que participaram do estudo, a predominância foi do sexo feminino $(51,58 \%)$ e a idade média foi de 7,99 $\pm 3,23$ anos. Das amostras analisadas 5,79\% eram positivas para parasitas, sendo 3,16\% de cistos de Entamoeba coli, 1,58\% Endolimax nana e 1,05\% Giardia lamblia. Sobre os conhecimentos relacionado a parasitas, $73,16 \%$ relataram saber da existência dos parasitas e o que eles causam no indivíduo e $68,42 \%$ conhecem os meios de transmissão, porém $41,58 \%$ consideram insuficientes as informações disponíveis para a população. Referente às condições sanitárias as quais estão expostos, 94,21\% consomem água potável e $64,74 \%$ afirmaram ter coleta e tratamento de esgoto. O presente estudo teve baixa frequência de parasitoses, isso pode ser explicado pelo conhecimento dos responsáveis, pelas condições sanitárias favoráveis, e pelo alto percentual $(78,95 \%)$ de escolares que já fizeram uso de antiparasitários.
\end{abstract}

Palavras chave: Estudantes. Infecções por protozoários. Perfis sanitários.

\begin{abstract}
The present study assess the frequency of intestinal parasites in São Marcos city-RS elementary and middle school children, from March to October 2015. A questionnaire was applied to asses students' knowledge on pathways for parasite transmission and on sanitation facilities in the city. Stool samples were collected from eighth-grade students of nine different schools and were analyzed/processed by spontaneous sedimentation. Of the 190 students who participated in the study, most were female $(51.58 \%)$ and the mean age of the students was $7.99 \pm 3.23$ years. $5.79 \%$ of the samples were positive for parasites, being 3.16\% of cysts of Entamoeba coli, $1.58 \%$ of Endolimax nana and $1.05 \%$ of Giardia lamblia. As for the students' knowledge on parasites, $73.16 \%$ reported knowing what parasites are and what damage they can cause, $68.42 \%$ are aware of the transmission pathways, however $41.58 \%$ consider there is insufficient information available to the public. Related to the health issues to which they are exposed, $94.21 \%$ reported consuming potable water and $64.74 \%$ reported having sewage collection and treatment. The present study has shown a low prevalence of parasitic infections, which could be

\footnotetext{
${ }^{1}$ Biomédica pela Faculdade da Serra Gaúcha. E-mail: scheilacristina@outlook.com.

${ }^{2}$ Biomédica pela Faculdade da Serra Gaúcha, mestranda em Biotecnologia pela Universidade de Caxias do Sul. Analista no Laboratório Lavin. E-mail: nati_cavagnolli@hotmail.com.

${ }^{3}$ Bióloga, mestre e doutora em Biotecnologia pela Universidade de Caxias do Sul. Docente da Faculdade da Serra Gaúcha. E-mail: patricia.spada@fsg.br.

${ }^{4}$ Biomédica pela Universidade Feevale, mestre em Biotecnologia pela Universidade de Caxias do Sul. Pesquisadora no Laboratório Alfa LTDA. E-mail: adry.dr@gmail.com.
} 
explained by the knowledge of parents and guardians about topic, favorable sanitary conditions, and the high percentage $(78.95 \%)$ of students who have already been treated with antiparasitic drugs.

Keywords: Students. Protozoal infections. Health profiles.

\section{Introdução}

As infecções parasitárias são consideradas um dos maiores problemas do mundo em saúde pública, principalmente em países em desenvolvimento (BASSO et al., 2008). A ausência ou insuficiência de saneamento básico, de higiene pessoal e doméstica são consideradas as principais causas da transmissão dos parasitas intestinais (PRADO et al., 2001). Os parasitas se adaptam muito bem em hospedeiros expostos a condições de saneamento básico precárias, baixo grau de escolaridade e hábitos de higiene inadequados (BASSO et al., 2008). Considera-se que as doenças parasitárias são consequências e causas do subdesenvolvimento, acompanhadas do analfabetismo, alienação do povo e corrupção (NEVES, 2002).

Nota-se que escolares são um grupo de risco(BASSO et al., 2008) por estarem mais expostos a situações que aumentam as chances de infecções parasitárias, como por exemplo, por não possuírem hábitos adequados de higiene e estarem em contato constante com solo e água (SILVA et al., 2009). Os parasitas promovem efeitos agressivos diretos especialmente nesses hospedeiros, além de exercer importante influência sobre o estado nutricional, crescimento e função cognitiva (BRILHANTE et al., 2010). Entre tantos prejuízos trazidos pelas parasitoses intestinais, principalmente as de curso crônico e assintomáticas, podem ser citados: déficit de desenvolvimento físico e mental, incapacitação para o trabalho na população estudantil, resultando em baixo índice de aproveitamento escolar (BECKER et al., 2002). O diagnóstico desse tipo de infecção pode ser feito através de exames parasitológicos de fezes com intuito de identificar o parasita causador e posteriormente o médico optar pelo melhor tratamento (MAGALHÃES et al., 2013).

São Marcos é um município do Rio Grande do Sul, localizado na costa superior nordeste, $28^{\circ} 58$ min, latitude sul e $51^{\circ} 05$ min, longitude oeste, e tem uma estimativa de 20.191 habitantes segundo dados do IBGE em 2011 (INSTITUTO BRASLEIRO DE GEOGRAFIA E ESTATÍSTICA, 2015). A cidade possui 5 escolas municipais que atendem crianças e adolescentes da pré-escola a $8^{\mathrm{a}}$ série (ou primeiro a nono anos), e outras 5 escolas estaduais que também atendem alunos do ensino fundamental, totalizando uma média de 2.000 alunos (SÃO MARCOS, 2015). O objetivo do trabalho foi avaliar a frequência de parasitas intestinais nos estudantes de escolas públicas do município de São Marcos com a realização do exame parasitológico de fezes e relacionar com as condições socioeconômicas dos estudantes e de seus responsáveis.

\section{Materiais e Métodos}

Foi realizado um estudo transversal descritivo e analítico em estudantes de 9 escolas da cidade de São Marcos, Rio Grande do Sul, Brasil localizadas tanto em áreas urbanas quanto em áreas rurais, estando 7 das escolas localizadas na área central da cidade. São Marcos possui 5 escolas municipais e 5 escolas estaduais, todas foram convidadas a participar do projeto, mas uma escola estadual optou por não participar, pela dificuldade de realizar atividades com os alunos. O número amostral foi por conveniência, sendo que a coleta foi realizada entre os meses de junho a outubro de 2015. Os critérios de inclusão foram ser estudantes do ensino fundamental da rede pública de São Marcos, e ter preenchido e entregue o questionário junto com a amostra de fezes.

Visitou-se as escolas para apresentação do projeto e para convidá-las a participar do estudo. Foram enviados aos pais ou responsáveis, o termo de consentimento livre e esclarecido (TCLE), juntamente com um questionário para 
avaliação do conhecimento relacionado as parasitoses e as condições socioeconômicas em que vivem. O questionário utilizado foi validado por Cavagnolli et al. (2015). Foram entregues também instruções de coleta para amostra de fezes e o kit para coleta, que continha: um par de luvas, sacolas plásticas, recipiente para coleta e uma etiqueta para identificação da amostra. As amostras foram refrigeradas pelos pesquisadores por no máximo 24 horas até o processamento. Não foi utilizado nenhum tipo de conservante no frasco de coleta, para evitar qualquer tipo de interferente, já que alguns conservantes podem alterar as estruturas parasitárias.

As amostras de fezes foram processadas pelo método de sedimentação espontânea de Hoffman, Pons e Janer (HPJ), a qual possibilita a concentração e identificação de cistos e oocistos de protozoários e, ovos e larvas de helmintos, além ser de fácil execução e de baixo custo (DE CARLI, 2008). Após o período mínimo de 2 horas de sedimentação, os sedimentos com lugol entre lâmina e lamínula foram analisados microscopicamente por dois observadores no Laboratório de Parasitologia da Faculdade da Serra Gaúcha. Após, os laudos das amostras positivas foram avaliados por um médico clínico que receitou a medicação Albendazol®, 2 frascos. A medicação foi disponibilizada pela secretaria de saúde do município. Os laudos foram entregues a cada participante, e os que apresentaram amostras positivas, já receberam também a medicação.

Os dados foram expressos em médias, percentagens e desvio-padrão, conforme melhor adequação. Para a análise estatística foi realizado o teste de q-quadrado com níveis de significância de $p<0,05$, utilizando o software SPSS 20.0 para Windows. O presente estudo foi aprovado pelo CEP Círculo Operário/FSG, sob parecer 479.964.

\section{Resultados}

Dos 228 escolares participantes do estudo, com uma perda amostral de 38 , por falta de amostra ou questionário. Entre os 190 escolares incluídos, a predominância foi do sexo feminino $(n=98$; $51,6 \%$ ), e a idade média foi de $7,99 \pm 3$ anos. A idade média dos responsáveis foi de 27,5 $\pm 18,73$ anos, e a maioria também do sexo feminino $(n=155 ; 81,6 \%)$. Com relação a escolaridade dos entrevistados, $40 \%$ possui ensino fundamental incompleto, conforme dados descritos na Tabela 1.

Sobre as questões de conhecimento dos responsáveis referente as parasitoses, 73,2\% relatam saber o que são parasitas e $68,4 \%$ dizem conhecer os meios de transmissão. Os responsáveis afirmam obter as informações por meio de profissionais da saúde $50,5 \%$, seguido por fontes como a televisão $22,6 \%$ e internet $8,9 \%$. Destes $53,2 \%$ consideram suficientes as informações disponíveis para a população (Tabela 1).

Observou-se que $38,4 \%$ dos estudantes não fazem exames de fezes antes ou depois da ingestão da medicação antiparasitária, entretanto $78,9 \%$ já fizeram uso de vermífugo, sendo $46,8 \%$ uma vez por ano. Um total de $22,1 \%$ dos responsáveis adquire a medicação para tratamento da criança quando necessário, sem prescrição médica. A maioria dos responsáveis $(56,3 \%)$ não informou o nome da medicação usada, e 26,3\% relataram utilizar a marca Albendazol ${ }^{\circledR}$ para tratamento parasitário.

Quanto as condições de saneamento básico da população estudada, 94,21\% consomem água potável, $64,74 \%$ possuem coleta e tratamento de esgoto e $94,21 \%$ afirmaram ter coleta de lixo (Tabela 2). A captação de água das chuvas por meio de bueiros é realizada em $65,26 \%$ das localidades da cidade. Sobre pragas, ratos e insetos, 53,68\% afirmam ter controle das mesmas na cidade, porém $37,37 \%$ dizem que existe a presença dessas pragas no município. Das amostras de fezes coletadas pelos estudantes, obteve-se presença apenas de protozoários, sendo cistos de Entamoeba coli (3,16\%), Endolimax nana (1,58\%) e Giardia lamblia (1,05\%) (Tabela 2). Não houve associação estatística significativa entre os resultados positivos e as variáveis, sexo $(p=0,258)$, idade dos estudantes 
$(\mathrm{p}=0,754)$, série atual dos participantes $(\mathrm{p}=0,067)$ resultado esse que pode ser explicado, ao menos em e uso de tratamento antiparasitário $(\mathrm{p}=0,398)$, parte, pelo número reduzido de amostras.

Tabela 1 - Informações gerais sobre o conhecimento de parasitoses pelos responsáveis dos escolares, diagnóstico e tratamento de parasitoses em escolares de São Marcos, Rio Grande do Sul, Brasil, obtidos por meio da aplicação de questionário em 2015

\begin{tabular}{|c|c|c|c|}
\hline & Variável & $\mathbf{n}$ & $\begin{array}{c}\text { Porcentagem } \\
(\%)\end{array}$ \\
\hline \multirow{7}{*}{ Escolaridade dos responsáveis } & Fundamental incompleto & 76 & 40,00 \\
\hline & Fundamental completo & 19 & 10,00 \\
\hline & Médio incompleto & 06 & 3,16 \\
\hline & Médio completo & 57 & 30,00 \\
\hline & Superior incompleto & 8 & 4,21 \\
\hline & Superior completo & 8 & 4,21 \\
\hline & Não informado & 16 & 8,42 \\
\hline \multirow{3}{*}{ Os responsáveis sabem o que são parasitoses? } & Sim & 139 & 76,16 \\
\hline & Não & 47 & 24,74 \\
\hline & Não Informado & 4 & 2,11 \\
\hline \multirow{3}{*}{$\begin{array}{l}\text { Os responsáveis têm conhecimento sobre os } \\
\text { meios de transmissão de parasitos? }\end{array}$} & Sim & 130 & 68,42 \\
\hline & Não & 55 & 28,95 \\
\hline & Não Informado & 5 & 2,63 \\
\hline \multirow{7}{*}{$\begin{array}{l}\text { Onde os responsáveis obtêm informações sobre } \\
\text { parasitos? }\end{array}$} & Profissionais da saúde & 96 & 50,53 \\
\hline & Televisão & 43 & 22,63 \\
\hline & Internet & 17 & 8,95 \\
\hline & Rádio & 8 & 4,21 \\
\hline & Jornal & 7 & 3,68 \\
\hline & Outros & 6 & 3,16 \\
\hline & Não informado & 13 & 6,84 \\
\hline \multirow{3}{*}{$\begin{array}{l}\text { Os responsáveis julgam suficientes as } \\
\text { informações disponíveis sobre parasitos? }\end{array}$} & Sim & 101 & 53,20 \\
\hline & Não & 79 & 41,58 \\
\hline & Não informado & 10 & 5,26 \\
\hline \multirow{3}{*}{$\begin{array}{c}\text { A criança já recebeu tratamento para } \\
\text { parasitoses? }\end{array}$} & Sim & 150 & 78,95 \\
\hline & Não & 35 & 18,42 \\
\hline & Não informado & 5 & 2,63 \\
\hline \multirow{4}{*}{$\begin{array}{l}\text { Frequência que a criança faz uso de } \\
\text { antiparasitários }\end{array}$} & Anualmente & 89 & 46,84 \\
\hline & Semestralmente & 36 & 18,95 \\
\hline & Quando necessário & 2 & 1,05 \\
\hline & Não informado & 63 & 33,16 \\
\hline \multirow{6}{*}{$\begin{array}{l}\text { Marcas de medicamentos citados para o } \\
\text { tratamento de parasitoses }\end{array}$} & Albendazol ${ }^{\circledR}$ & 50 & 26,32 \\
\hline & Annita ${ }^{\circledR}$ & 9 & 4,74 \\
\hline & Pontenium $\AA$ & 1 & 0,53 \\
\hline & Parasin $\AA$ & 5 & 2,63 \\
\hline & Não lembra & 18 & 9,47 \\
\hline & Não informado & 107 & 56,32 \\
\hline
\end{tabular}

Fonte: Autores. 
Tabela 2- Dados do saneamento básico das residências e frequência de parasitas em escolares de São Marcos, Rio Grande do Sul, Brasil, em 2015.

\begin{tabular}{ccccc}
\hline Variáveis sanitárias & Sim (\%) & Não (\%) & Não informado (\%) & p valor* \\
\hline Água potável e encanada & 94,21 & 4,21 & 1,58 & 0,164 \\
Coleta e tratamento de esgoto & 64,74 & 32,11 & 3,15 & 0,996 \\
Ruas e proximidades limpas & 78,42 & 17,89 & 3,69 & 0,981 \\
Boa captação da água das chuvas & 65,26 & 31,05 & 3,69 & 0,205 \\
Coleta de lixo & 94,21 & 4,21 & 1,58 & 0,088 \\
Controle de pragas (ratos e insetos) & 53,68 & 43,68 & 2,64 & 0,074 \\
Presença de pragas (ratos e insetos) & 37,37 & 58,42 & 4,21 & 0,159 \\
\hline Resultados das análises & $\mathbf{N}$ & $\mathbf{\%}$ & & \\
parasitológicas das fezes & & & \\
Negativos & 179 & 94,21 & & \\
Entamoeba coli & 6 & 3,16 & & \\
Endolimax nana & 3 & 1,58 & & \\
Giardia lamblia & 2 & 1,05 & & \\
\hline
\end{tabular}

*Valores de $p$ para análise de associação entre a positividade dos resultados e as variáveis descritas, pelo teste de q-quadrado.

Fonte: Autores.

\section{Discussão}

As parasitoses constituem um problema de saúde pública no mundo. O presente estudo obteve um baixo percentual de amostras positivas (5,79\%) nos escolares de São Marcos, o que se difere de outras pesquisas realizadas no Brasil. Na cidade de Caxias do Sul, município vizinho de São Marcos, em um estudo realizado no período de 1969 a 2004, que também avaliou resultados de exames parasitológico de fezes de escolares por sedimentação espontânea, teve maior prevalência de amostras positivas (58\%), sendo $47 \%$ de Ascaris lumbricoides, $36 \%$ de Trichuris trichuria, $8 \%$ de Enterobius vermiculares, e os protozoários encontrados foram $24 \%$ de Giardia lamblia, e $20 \%$ de Entamoeba coli. No ano de 1969 a prevalência total de parasitoses, especialmente de helmintos, foi maior do que em 2004, isso pode ser explicado pela melhoria da infraestrutura e saneamento básico realizados na cidade com passar dos anos (BASSO et al., 2008). Esse estudo assim como o nosso não obteve diferença significativa de parasitados entre zona urbana e rural. Outros estudos com prevalência maior de parasitoses do que o nosso são: em Sete Lagoas-MG com 34,6\% de casos positivos (PIRES et al., 2016) e na região sul do Brasil foi em UbiratãPR com 24,56\% de amostras positivas (MIOTTO et al., 2016), São Joaquim-SC com 22,36\% (SCHMITT; PAES, 1997), em Xanxerê-SC com 66\% e 19 parasitos diferentes observados (COSTA et al., 2015), em Novo Hamburgo (cidade localizada no Rio Grande do Sul) com 53,85\%, onde destacase a prevalência maior para o helminto Ascaris lumbricoides (25,70\%), e entre os protozoários, de Giardia lamblia (22,90\%) (BECKER et al., 2002).

Já no estudo realizado em Estiva Gerbi no estado de São Paulo (ANDRADE; FERREIRA, 2005), os autores obtiveram uma prevalência de 11,5\% nos escolares, e em São Miguel do Oeste-SC, 7,4\% de amostras positiva (SEGER et al., 2010), sendo resultados mais próximos aos encontrados em nosso estudo. Assim como o estudo realizado em Flores da Cunha (CAVAGNOLLI et al., 2015) e Caxias do Sul (CAMELLO et al., 2016) (municípios próximos 
a São Marcos), que tiveram um percentual de $10 \%$ e 5,8\% de amostras positivas respectivamente. No primeiro estudo $55,9 \%$ foram de cistos de Endolimax nana, 26,5\% de Entamoeba coli, 5,9\% de Iodamoeba butschlii, 2,9\% de Giardia lamblia, 2,9\% de ovos de Ascaris lumbricoides e 5,9\% de parasitose múltipla (Entamoeba coli e Endolimax nana) e no segundo estudo, foram observados apenas protozoários sendo $60 \%$ cistos de Endolimax nana, 26,7\% de Entamoeba coli e 13,3\% de Giardia lamblia. No nosso estudo também foram encontrados somente protozoários, sendo dentre as amostras positivas, 54,55\% de Entamoeba coli, $27,27 \%$ de Endolimax nana e 18,18\% de Giardia lamblia. O estudo realizado em Belo Horizonte$\mathrm{MG}$, teve resultados ainda mais semelhantes ao nosso, pois também encontraram somente presença de protozoários nas amostras analisadas, sendo Giardia lamblia (33,3\%), Entamoeba coli $(33,3 \%)$ e Endolimax nana (33,3\%) (SEMIÃO et al., 2015). Um estudo realizado no Paraná, também apresentou amostras positivas para protozoários, sendo eles, Endolimax nana (6,5\%), Entamoeba coli (3,5\%), Giardia lamblia (6,3\%), resultados semelhantes ao nosso estudo, e diferenciando-se pela presença de helmintos, como: Ascaris lumbricoides (1,4\%), Strongyloides stercoralis (0,7\%), Enterobius vermicularis $(0,7 \%)$, Ancilostomídeo $(0,2 \%) \quad e$ Taenia sp (0,2\%) (SANTOS; MERLINI, 2010).

As condições de vida precária, higiene inadequada, más condições no abastecimento de água, entre outros, são fatores determinantes para transmissão de parasitas e desenvolvimento da doença (LUDWIG et al., 1999). Quanto ao saneamento básico, um estudo realizado no Ceará mostrou que somente $38 \%$ das moradias consumiam água tratada, e dos 383 indivíduos avaliados, 233 apresentavam resultado positivo para o exame parasitológico de fezes (VASCONCELOS et al., 2011). Um estudo realizado em uma comunidade indigena Mbyá-Guarani, em Porto Alegre (cidade também localizada no Rio Grande do Sul) que apresentou ausência de infra-estrutura destinada ao recolhimento de resíduos humanos e animais doméstico, e a inexistência de água potável, apresentou um alto percentual $(88,7 \%)$ de parasitas, devido a todos os fatores inadequados de saneamento básico que envolviam a população estudada (BRANDELLI et al., 2012). Em comparação aos resultados encontrados no nosso estudo, 94,21\% consomem água potável, $64,74 \%$ possuem coleta e tratamento de esgoto, o que pode justificar a baixa frequência de parasitoses $(5,79 \%)$, devido as boas condições de saneamento básico do município. O projeto de saneamento básico da cidade foi criado em 2013 e prevê avaliação e cumprimentos das metas a cada quatro anos (SÃO MARCOS, 2015).

Nosso estudo se difere da maior parte das pesquisas citadas acima, pois apresentou resultados de análise de fezes somente com protozoários. Isso pode ser explicado, além das condições de saneamento básico, pelo fato de ter sido realizada somente uma técnica (sedimentação espontânea), que embora detecte cistos, ovos e larvas, há metodologias mais específicas para identificação de diferentes estruturas parasitárias, tendo isso como uma limitação. No estudo de Brandelli et al. (2012) que foram realizadas três técnicas: por sedimentação espontânea, centrifugação-flutuação e Kato-Katz a prevalência de enteroparasitoses foi alta $(88,7 \%)$, embora as condições sanitárias também sejam diferentes. Outra limitação do estudo que pode ser citada, é a coleta única de amostra de fezes, onde sabe-se que quanto maior o número amostral, maior as chances de encontro de estruturas parasitárias, visto que, muitos parasitas são liberados de forma intermitente nas fezes (DE CARLI, 2008). Em vista das situações expostas, mais estudos se fazem necessários para confirmação e melhor avaliação da população em questão.

No Brasil, a prevalência de parasitas prevalece nas regiões tropicais e subtropicais, pois temperaturas elevadas e tempo úmido criam condições ideais para que o ciclo dos parasitas se complete (SANTOS; WIEBBELING; MEZZARI, 2003). Outro fator que pode ter influência é o nível 
de educação da população em pesquisa, onde no nosso estudo $30 \%$ dos responsáveis possui ensino médio completo resultado semelhante ao estudo de Cavagnolli et al. (2015) e Camello et al. (2016), que mostraram que $23,1 \%$ e $28,4 \%$ dos responsáveis, respectivamente, possuem ensino médio completo. Percebeu-se que a população de São Marcos, $76,16 \%$ tem conhecimento sobre parasitoses, 68,42 \% afirmam conhecer os meios de transmissão, e 53,2 consideram as informações suficientes. E essas informações são dadas através de profissionais da saúde $(50,53 \%)$, pela televisão $(22,63 \%)$ e pela internet $(8,95 \%)$, resultados semelhantes também ao estudo de Cavagnolli et al. (2015), que apresentou um percentual de $75,7 \%$ para conhecimentos sobre os parasitas, $67,2 \%$ conhecem os meios de transmissão, e a maioria $(49,7 \%)$ obtém as informações através de profissionais da saúde.

Sobre o uso de medicação antiparasitária, $78,95 \%$ já fizeram uso de vermífugos, sendo que $22,11 \%$ adquirem medicação sem prescrição médica, percentual semelhante ao encontrado no estudo de Flores da Cunha, onde 28,7\% também fazem uso de medicação sem prescrição médica (CAVAGNOLLI et al., 2015), o que comprova que existe automedicação indiscriminada.

Conclui-se com o presente estudo que a cidade de São Marcos felizmente possuí uma baixa frequência de parasitoses, e isso se deve as boas condições de saneamento básico, ao abastecimento de água potável, ao nível de conhecimento dos responsáveis sobre parasitoses e, pelo fator não tão positivo, que é o uso de medicação antiparasitária sem realização de exame parasitológico de fezes e acompanhamento médico. É indispensável a realização de mais campanhas de conscientização e informação nas escolas, e na população em geral, para acrescentar conhecimentos sobre a automedicação, parasitoses e como evitar possíveis infecções. O setor da saúde é falho muitas vezes, pela falta de planejamento de programas e campanhas de conscientização sobre o assunto.

\section{Referências}

ANDRADE, C. S.; FERREIRA, G. R. Alguns aspectos socioeconômicos relacionados a parasitoses intestinais e avaliação de uma intervenção educativa em escolares de Estiva Gerbi, SP. Revista da Sociedade Brasileira de Medicina Tropical, Uberaba, v. 38, n. 5, p. 402405, 2005.

BASSO, R. M. C; SILVA-RIBEIRO, R. T.; SOLIGO, D. S; RIBACKI, S. I; CALLEGARIJACQUES, S. M.; ZOAS, B. C. A. Evolução da prevalência de parasitoses intestinais em escolares em Caxias do Sul, RS. Revista da Sociedade Brasileira de Medicina Tropical, Uberaba, v. 41, n. 3, p. 263-268, 2008.

BECKER, A. A.; LOSCHPE, R.; DELWING, D.; DELWING, D.; CANALI, J. Incidência de parasitoses intestinais em escolares do município de Novo Hamburgo-RS. Revista Brasileira de Análises Clinica, Rio de Janeiro, v. 34, n. 2, p. 85-87, 2002.

BRANDELLI, C. L. C.; DE CARLI, G. A.; MACEDO, A. J.; TASCA, T. Intestinal parasitism and socio-environmental factors among MbyáGuarani Indians, Porto Alegre, Rio Grande do Sul, Brazil. Revista Instituto Medicina Tropical de São Paulo, São Paulo, v. 54, n. 3, p. 119-22, 2012.

BRILHANTE, A. F.; KNECHTEL, D. S.; NUNES, V. L. B.; GOMES, P. D. M. F. Enteroparasitoses em escolares do distrito águas do Miranda, município de Bonito, Mato Grosso do Sul. Revista de Patologia Tropical, Goiânia, v. 39, n. 4, p. 299-307, 2010.

CAMELLO, J. T.; CAVAGNOLLI, N. I.; SPADA, P. W. D. S.; POETA, J.; RODRIGUES, D. R. Prevalência de parasitoses intestinais e condições de saneamento básico das moradias de escolares da zona urbana de Caxias do Sul, Rio Grande do Sul. Scentia Medica, Porto Alegre, v. 26, n. 1, p. 21716, 2016.

CAVAGNOLLI, N. I.; CAMELLO, J. T; TESSER, S.; POETA, J.; RODRIGUES, A. D. Prevalência de enteroparasitoses e análise socioeconômicas de escolares em Flores da Cunha-RS. Revista Patologia Tropical, Goiânia, v. 44, n. 3, p. 312-322, 2015. 
COSTA, T. D.; DE ANDRADE, D. F. R.; BARROS, V. C.; DE FREITAS, D. R. J. Análise de enteroparasitoses em crianças em idade pré-escolar em município de Santa Catarina, Brasil. Revista Prevenção de Infecção e Saúde, Teresina, v. 1, n. 2, p. 1-9, 2015.

DE CARLI, G. A. Parasitologia clínica. 2. ed. São Paulo: Atheneu, 2008.

IBGE. Disponível online em: <www.ibge.com.br> Acesso em: 18 jul. 2015.

LUDWIG, K. M.; FREI, F.; ALVARES FILHO, F.; RIBEIRO-PAES, J. T. Correlação entre condições de saneamento básico e parasitoses intestinais na população de Assis, Estado de São Paulo. Revista Sociedade Brasileira de Medicina Tropical, Uberaba, v. 32, n. 5, p. 547-555, 1999.

MAGALHÃES, R. F.; AMARO, P. F.; SOARES, E. B.; LOPES, L. A.; MAFRA, R. S. P.; ALBERTI, L. R. Ocorrência de enteroparasitoses em crianças de creches na região do Vale do Aço-MG. Unopar Científica: Ciências Biológicas e da Saúde, Londrina, v. 15, n. 3, p. 187-91, 2013.

MIOTTO, J. E.; CARO, D. S. A.; DE BARROS, M. F.; REGO, B. E. F.; DOS SANTOS, F. C.; MACAGNAN, R.; SANTOS, I. S. Diagnóstico laboratorial de enteroparasitosese anemia e sua possível associação com eosinofilia em crianças em idade escolar em Ubiratã-PR. Biosaúde, Londrina, v. 16, n. 2, p. 52-62, 2016.

NEVES, D. P. Parasitologia humana. 10. ed. São Paulo: Atheneu, 2002.

PIRES, E. D. C. R.; GUIMARÃES, F. P.; CARDOSO, A. K. O. S.; SANTOS, R. R.; LIMA, K. A. S.; TRUOCCHIO, M. R. Estudo preliminar das parasitoses intestinais em escolares da microrregião de Sete Lagoas-Mg. Revista Brasileira de Ciências da Vida, Sete Lagoas, v. 3, n. 2, p. 1-4, 2016.

PRADO, M. S.; BARRETO, M. L.; STRINA, A.; FARIA, J. A. S.; NOBRE, A. A.; JESUS, S. R. Prevalência e intensidade da infecção por parasitas intestinais em crianças na idade escolar na cidade de Salvador, Bahia, Brasil. Revista Sociedade Brasileira de Medicina Tropical, Uberaba, v. 34, n. 1, 2001.
SANTOS, D. E.; WIEBBELING, A. M. P.; MEZZARI, A. Parasitos intestinais: aspectos gerais e prevalência em uma escola da periferia de Porto Alegre-RS, Revista Newslab, São Paulo, v. 60, p. 118-134, 2003.

SANTOS, S. A.; MERLINI, L. S. Prevalência de enteroparasitoses na população do município de Maria Helena, Paraná State. Ciência e Saúde Coletiva, Rio de Janeiro, v. 15, n. 3, p. 899-905, 2010.

SÃO MARCOS. Prefeitura Municipal. Disponível em: <www.saomarcos.rs.gov.br>. Acesso em: 23 abr. 2015.

SCHMITT, M. L.; PAES, M. A. S.; Prevalência de parasitoses intestinais em escolares no município de São Joaquim, SC. Revista Brasileira de Análises Clínicas, Rio de Janeiro, v. 29, n. 4, p. 215-6, 1997.

SEGER, J.; SOUZA, W. M.; MARANGONI, J. C. F.; MASCHIO, V. J.; CHIELLI, E. O. Prevalência de parasitas intestinais na população do Bairro Salete, município de São Miguel do Oeste, SC. Unoesc \& Ciência: ACBS, Joaçaba, v. 1, n. 1, p. 53-56, 2010.

SEMIÃO, C. M.; FERNANDES, D. L. M.; COELHO, R. O. V.; BELÉM, M. E. P.; ROCHA, R. D. R.; GONÇALVEZ, G. S. Prevalência de parasitoses intestinais em crianças de uma creche do município de Belo Horizonte, Minas Gerais. Revista Iniciação Cientifica Newton, São Paulo, n. 2, 2015.

SILVA, E. F.; SILVA, E. B.; ALMEIDA, K. S.; SOUZA, J.J.N.;FREITAS, F.L.C. Enteroparasitoses em crianças de áreas rurais do município de Coari, Amazonas, Brasil. Revista de Patologia Tropical, Goiânia, v. 38, p. 355-363, 2009.

VASCONCELOS, I. A. B.; OLIVEIRA, J. W.; CABRAL, F. R. F.; COUTINHO, H. D. M.; MENEZES, I. R. A. Prevalência de parasitoses intestinais entre crianças de 4-12 anos no Crato, Estado do Ceará: um problema recorrente de saúde pública. Acta Scientiarium: Health Science, Maringá, v. 33, n. 1, p. 35-41, 2011.

Recebido em: 14 dez. 2015. Aceito em: 05 jun. 2016. 